\title{
Combinación de una metodología basada en proyectos y de una evaluación competitiva en la asignatura Análisis y modelación de redes de distribución de agua
}

\section{Pedro L. Iglesias-Rey y F. Javier Martínez-Solano ${ }^{\mathrm{b}}$}

${ }^{a}$ Dpto. Ingeniería Hidráulica y Medio Ambiente. Universitat Poltècnica de València. email: piglesia@upv.es, 'DDto. Ingeniería Hidráulica y Medio Ambiente. Universitat Poltècnica de València. email: jmsolano@upv.es.

\begin{abstract}
One of the challenges arising from the implementation of the European Higher Education Area is the search for new methods of evaluation and learning. This paper proposes a new project-based learning initiative and a competitive evaluation system in the student's knowledge related to the analysis and planning of water distribution networks. The methodology is based on the development of a team work project to improve an existing water supply network. Based on computer simulations and acquired learning, students must find the best solution for system operation. Finally, the works (presented and exposed) are evaluated in a competitive way, giving the highest score to the best solution according to previously established criteria. This initiative has been implemented in the subject "Analysis and modeling of water distribution networks" that is taught in the Master in Hydraulic Engineering and Environment of the UPV. The initial results show a better perception of the subject by the students and a certain improvement in their academic results.
\end{abstract}

Keywords: project-based learning, competitive evaluation, team work, water distribution networks

\begin{abstract}
Resumen
Uno de retos derivados de la puesta en marcha del Espacio Europeo de Educación Superior es la búsqueda de nuevos métodos de evaluación y aprendizaje. En este trabajo se plantea una nueva iniciativa de aprendizaje basada en proyectos y en una evaluación competitiva de los alumnos en sus conocimientos relacionados con el análisis y planificación de las redes de distribución de agua. La metodología se basa en el desarrollo en grupo de un proyecto de mejora de una red de abastecimiento existente. A partir de simulaciones computacionales y de los conocimientos adquiridos los alumnos deben encontrar la mejor solución de funcionamiento del sistema. Finalmente los trabajos presentados y expuestos son valorados de forma competitiva otorgando la máxima puntuación a la mejor solución de acuerdo a unos criterios previamente establecidos. Esta iniciativa ha sido implementada en la asignatura "Análisis y modelación de redes de distribución de agua" que se imparte en el Máster en Ingeniería Hidráulica y Medio Ambiente de la UPV. Los resultados iniciales muestran una mejor
\end{abstract}


percepción de la asignatura por parte de los alumnos y una cierta mejora en los resultados académicos de los mismos.

Palabras clave: aprendizaje basado en proyectos, evaluación competitiva, trabajo en grupo, redes de distribución de agua

\section{Introducción}

Desde un punto de vista estrictamente profesional es cada día más evidente la la preocupación por la docencia de los futuros ingenieros. Así diferentes organizaciones empresariales (Henry, 2002) se han preocupado por los retos profesionales de la ingeniería civil ante el nuevo milenio. Tras varios estudios preliminares y encuestas a diferentes miembros de la American Society of Civil Engineering (ASCE) se enunciaron 6 retos fundamentales:

- Concienciación pública sobre el papel de la ingeniería civil en las infraestructuras.

- Interacción de los ingenieros civiles con la sociedad: su relación con los cargos públicos, la política y el servicio a la comunidad.

- El máster: primer título directamente relacionado con la actividad profesional.

- Recuperar el rol de liderazgo lo relacionado con el desarrollo de infraestructuras.

- El impacto del diseño constructivo en los profesionales de la ingeniería civil.

- La competitividad del precio de las soluciones.

Este interés en dar a la ingeniería civil un enfoque práctico (Luce y Henry, 2002) ha originado que en los sistemas escolares y principalmente en las carreras de ingeniería sea cada día más necesario diseñar y emplear juegos basados en simulaciones del ejercicio profesional. De este tipo es el bien conocido concurso de diseño de puentes realizado durante más de 15 años por ASCE y la United States Militaty Academy (USMA).

La mejora de los sistemas de aprendizaje y evaluación es una de las líneas de trabajo en las que se centran todas las innovaciones educativas desarrolladas a partir de la puesta en marcha del Espacio Europeo de Educación Superior (EEES). Los cambios acaecidos en la universidad española a raíz de la entrada del EEES (Espacio Europeo de Educación Superior) han originado la necesidad de actualizar gran parte de las metodologías docentes utilizadas (Caro et al., 2008). Una de las iniciativas de este EEES es aumentar la motivación del alumnado. Es decir, se hace necesario transformar de forma continua el método de aprendizaje para garantizar una continua atención por parte de los estudiantes (Álvarez et al., 2008).

Sin duda una de las mejores formas de cambiar la actitud del alumnado respecto de una materia es introducir novedades tanto en la metodología desarrollada como en el método de evaluación empleado. Es por ello que en este trabajo se analiza la influencia que puede tener el cambio de una metodología de evaluación y de trabajo en la percepción e interés de los alumnos sobre la materia. 
En la literatura científica existen multitud de referencias a nuevas experiencias relacionadas con metodologías de aprendizaje y de evaluación innovadoras, en las cuales la presencia del ordenador forma parte del día a día. Así Merino y Abel (2003) demuestran en sus experimentos que un método de aprendizaje basado en ordenador puede ser alternativo a los modelos clásicos de aprendizaje. En esta línea, Chumbley et al. (2002) presentan la experiencia de una plataforma web desarrollada para complementar la formación de determinados alumnos en clase.

Sin embargo, la idea no es sólo introducir el ordenador o las herramientas de simulación, sino acercar el desarrollo de nuestros ingenieros a lo que será su futura vida profesional. Así Bourgault et al. (2002) presentan un enfoque pedagógico consistente en recrear el contexto en el cual deben desenvolverse los ingenieros a la hora de gestionar sus proyectos. De hecho, al igual que en la mayoría de proyectos este enfoque educativo requiere de soluciones tecnológicas innovadoras y hace imprescindible desarrollar habilidades para la coordinación del trabajo en grupo (Slavin y Johnson, 1999). Más aún, las innovaciones en la tarea educativa pueden alcanzar la evaluación. Así, una experiencia alternativa (Romero et al., 2009) en la que se evalúa a los estudiantes a través del uso de teléfonos en lugar de evaluaciones basadas en páginas web permite aumentar el nivel de motivación de los alumnos.

\subsection{La utilización de los juegos y las herramientas de simulación como apoyo a la docencia}

Uno de de los elementos que más ha contribuido durante los últimos años al desarrollo de nuevas metodologías de aprendizaje es el auge que han experimentado los modelos de simulación prácticamente en todas las áreas del conocimiento. Así, la presencia de juegos o roles de simulación como parte de los métodos de aprendizaje (Mayer et al., 2014) no es algo nuevo. No es extraño encontrar soluciones en campos tan diversos como la electrónica (Zavalani, 2015), la gestión de proyectos (Davidovich et al., 2006) o la ingeniería hidráulica (Morley et al., 2016).

En este contexto aparecen conceptos nuevos que pretenden definir las nuevas metodologías educativas disponibles. Expresiones como Serious Games (juegos serios o con contenido educativo) introducido por Abt (1970) o la idea de Simulation Gaming (juegos de simulacion) presentada por Duke (1974) han conducido finalmente a la definición de lo que se conoce como Ciencia del aprendizaje basado en el juego (Sánchez et al., 2010).

A partir de estas herramientas de simulación y estos juegos es posible desarrollar cursos completos de formación que utilicen estas herramientas de forma extensiva (Wu, 2012). De forma específica, en el campo de la asignatura objeto del presente de estudio cabe destacar las contribuciones de (Savic et al., 2016), quienes desarrollan una herramienta interactiva que permite el diseño, planificación y estudio de las redes de distribución de agua a través de un juego de simulación. Dicho juego presenta una interface web tras la cual se encuentra la herramienta de simulación EPANET (Rossman, 2000) de análisis de redes hidráulicas a presión. Más aún, su desarrollo se realizado con una filosofía de código abierto. Sin duda el 
disponer de referencias de código abierto o libre es una notable ventaja en el desarrollo de este tipo de metodologías, tal y como ponen de manifiesto Illie-Zudor et al. (2013).

\subsection{El aprendizaje basado en proyectos}

La utilización de técnicas educativas basadas en el desarrollo de proyectos en el área de la ingeniería civil es algo que viene realizándose desde hace tiempo (Mahendran, 1995). De forma más reciente (González-Marcos et al., 2014) utilizan ya este tipo de metodologías para evaluar el desarrollo de las competencias transversales que deben desarrollarse en cualquier titulación.

El aprendizaje basado en proyectos surge de la necesidad de exponer a los estudiantes a situaciones tales como: la necesidad de realizar procesos iterativos de diseño, la necesidad de formular hipótesis, de desarrollar soluciones, analizar o validar de estrategias. Todo ello incorporando experiencias propias y argumentos de la literatura científica. Por ello, este tipo de ejercicios son la oportunidad que los estudiantes tienen de inicializarse en la experiencia del diseño en proyectos. Este tipo de problemas además permite desarrollar otras habilidades relacionadas con la comunicación técnica (redacción de informes técnicos y presentación oral de soluciones y diseños) y con el trabajo en equipo (incluida la gestión de conflictos y la coordinación de equipos).

En este escenario existen experiencias previas en campos tan diversos como la simulación mediante modelos de elementos finitos (Ural, 2013), el desarrollo de algoritmos para el procesamiento de imágenes (García, 2013), el desarrollo de modelos de instrumentación virtual (See, 2006) utilizando la herramienta LabVIEW (Travis y Kring, 2006) o el diseño de células de fuel (Gadhamshetty et al., 2016).

En definitiva las experiencias analizadas son combinaciones entre el aprendizaje tradicional y el aprendizaje basado en proyectos. Un paso más allá es el trabajo realizado por De los Ríos et al. (2010), quienes proponen un proyecto basado en proyectos de tipo colaborativo. Este proyecto se enmarca dentro de los objetivos del EEES ya que la metodología empleada presenta respecto a casos anteriores tres ventajas principales: facilitar el entrenamiento en competencias de tipo personal, familiarizar al alumno con problemas de la práctica profesional (Clarke, 2007) e introducir a los estudiantes al aprendizaje colaborativo.

\subsection{La evaluación competitiva}

Existen muchos estudios sobre la evaluación del aprendizaje en la universidad, si bien uno de los más exhaustivos es el realizado por Valdivia (2008). En este trabajo realiza una profunda revisión bibliográfica sobre los métodos de evaluación del aprendizaje en la universidad. Esta revisión pretende resolver tres preguntas fundamentales: ¿cuáles son los principales problemas en la evaluación del aprendizaje en las universidades?, ¿son estos problemas similares o tienen manifestaciones diferentes en función del contexto? y ¿qué referencias científicas que permitan mejorar los métodos de evaluación? La discusión sobre este tipo de problemas es profunda, y existen multitud de referencias bibliográficas al respecto. 
En particular resulta de interés analizar los métodos de evaluación diferentes de los utilizados convencionalmente. Así, algunos de estos métodos serían: realización de trabajos, desarrollo de proyectos, confección de diarios, evaluaciones basadas en portafolio, desarrollo de mapas conceptuales o sistemas de evaluación por parte de los propios estudiantes, bien sea en forma de autoevaluación o en sistemas de evaluación por pares. En la Figura 1 se recoge la distribución de las diferentes técnicas de evaluación alternativa. Tal como puede apreciarse la evaluación basada en proyectos es todavía poco significativa.

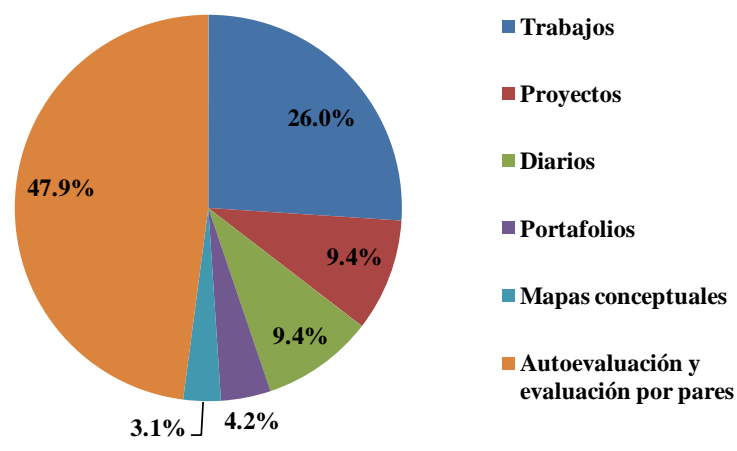

Figura 1. Porcentaje de métodos de evaluación alternativos (Valdivia, 2008).

Sin lugar a dudas las nuevas técnicas de evaluación juegan un papel crucial en el nuevo marco normativo derivado del EEES (Hernández-Linares et al., 2015). Así, aparecen experiencias en las que se fórmulas de evaluación alternativas Carpenter et al., (2007) proponen la utilización de ensayos técnicos para la evaluación en cursos de ingeniería, mientras que Baba et al., (2012) muestran como la lógica difusa puede ser utilizada para mejorar la calidad de los método educativos, la motivación y la fiabilidad y coherencia en los criterios de evaluación. Pero sin duda una de las formas de evaluación que últimamente ha tomado más auge es la basada en la competitividad de las soluciones de los alumnos ante problemas, proyectos o diseños planteados.

La competencia en diseño o en el desarrollo de proyectos es una aptitud que únicamente puede alcanzarse con un entrenamiento adecuado y a través de la acumulación de experiencia en el desarrollo de la misma. Uno de los caminos para proporcionar este tipo de competencias es a través de la resolución de proyectos complejos que finalmente requieran enfrentar entre sí las soluciones de los estudiantes (Labossière y Bisby, 2009). La competición entre estudiantes no es algo nuevo. Existen referencias (Wankat, 2005) que muestran la gran cantidad de competiciones entre estudiantes en el ámbito universitario. Algunas de estas competiciones cuentan con el apoyo de asociaciones profesionales tan importantes como la ASHRAE (American Society of Heating, Refrigeration and AirConditioning Engineers) o la AIChE (American Institute of Chemical Engineers). Sin embargo, este tipo de competiciones se centran más en la comparación entre los niveles de diferentes universidades.

Por el contrario, la idea de los métodos competitivos como herramienta de evaluación en nuestras universidades es algo diferente. Se centra en comparar entre sí las soluciones y resultados de los estudiantes que han tenido una misma formación: mismo temario, misma 
metodología docente, mismo profesor. Ejemplos estos métodos son los desarrollados por Adin Marcos et al., (2010), quien aplica esta metodología de evaluación para el diseño de circuitos electrónicos mediante el uso de herramientas CAD, o el propuesto por Shyr, (2012) en el campo de la ingeniería mecatrónica.

El trabajo en equipo es una parte esencial de la práctica de la ingeniería. Por lo general la educación universitaria centra su atención en el desarrollo de las habilidades técnicas de los estudiantes en actividades fundamentalmente centradas en el aula. Al mismo tiempo, se hace muy poco hincapié en el trabajo en equipo, a pesar de que esta habilidad es una de las más reconocidas para la actividad profesional de cualquier ingeniero. Por ello las competiciones grupales (Cerato et al., 2011) permiten complementar la enseñanza en clase y son fundamentales para aumentar las habilidades de trabajo en grupo. Es decir, las experiencias de evaluación competitiva deben combinarse con la organización de grupos de trabajo en clase (Çulça, 2016) o la presentación y defensa de los resultados (Beriain et al., 2014) frente a sus pares (sus compañeros). De esta forma aumenta la capacidad de auto aprendizaje de los alumnos (Schaffer et al, 2012), aumentan las capacidades de resolución de proyectos y mejoran la motivación hacia la materia objeto de estudio.

\section{Objetivos}

El objetivo de este trabajo es plantear un sistema de aprendizaje basado en proyectos en el campo de la hidráulica de sistemas de distribución de agua. Éste utilizará como técnica de evaluación un sistema de competitivo a partir de una serie de criterios previamente definidos. Finalmente el método se complementa con la elaboración de un informe técnico sobre la solución obtenida y la exposición oral de la misma. El campo de aplicación de la metodología a desarrollar será la asignatura Análisis y modelación de redes de distribución de agua (AMRDA) que se imparte en el Máster en ingeniería hidráulica y medio ambiente (MIHMA) de la Universidad Politécnica de Valencia (UPV).

De forma resumida el objetivo general del trabajo la mejora de la percepción por parte de los alumnos de la asignatura AMRDA a través del desarrollo de una metodología de aprendizaje basada en proyectos combinada con un sistema de evaluación competitivo. Este objetivo general se logrará en base al desarrollo de los siguientes objetivos específicos:

- Análisis previo de la motivación de los alumnos por la asignatura.

- Desarrollo de un método de aprendizaje basado en proyectos.

- Desarrollo de un método de evaluación competitivo para grupos de trabajo.

- Desarrollo de un método complementario a la evaluación competitiva.

- Análisis del impacto de la puesta en marcha del proyecto de innovación educativa.

En definitiva, la consecución de estos objetivos permitirá no solo mostrar la innovación educativa desarrollada, sino también ver los resultados de aplicación de la misma a lo largo del tiempo. 


\section{Desarrollo de la innovación}

\subsection{Descripción del tipo de proyecto}

El objeto central de la metodología docente planteada es el desarrollo de un proyecto de mejora de una red de abastecimiento de agua existente. Los antecedentes de este tipo de proyecto hay que encontrarlos en los desafíos sobre redes de abastecimiento de agua que eventualmente se realizan dentro de la comunidad científica (Walski et al., 1987; Ostfeld et al., 2008; Ostfeld et al., 2011; Marchi et al., 2013; Giustolisi et al., 2015) y que de forma genérica se han dado en llamar la Batalla de las Redes de Agua (The Battle of Water Networks). De hecho, los profesores de la asignatura tienen cierta experiencia en el desarrollo de este tipo de ejercicios (Iglesias et al, 2015).

El proyecto toma como punto de partida el modelo de una red de distribución de agua construido mediante el modelo EPANET (ver Figura 2). El funcionamiento de la red de forma general presenta algún tipo de deficiencia: insuficiencia de presiones, excesivo gasto energético en bombeo, gran cantidad de agua perdida por fugas, etc. A partir de dicho momento el objetivo del alumno es encontrar un grupo de trabajo (de máximo 3 personas) que de forma conjunta deben mejorar la red existente.

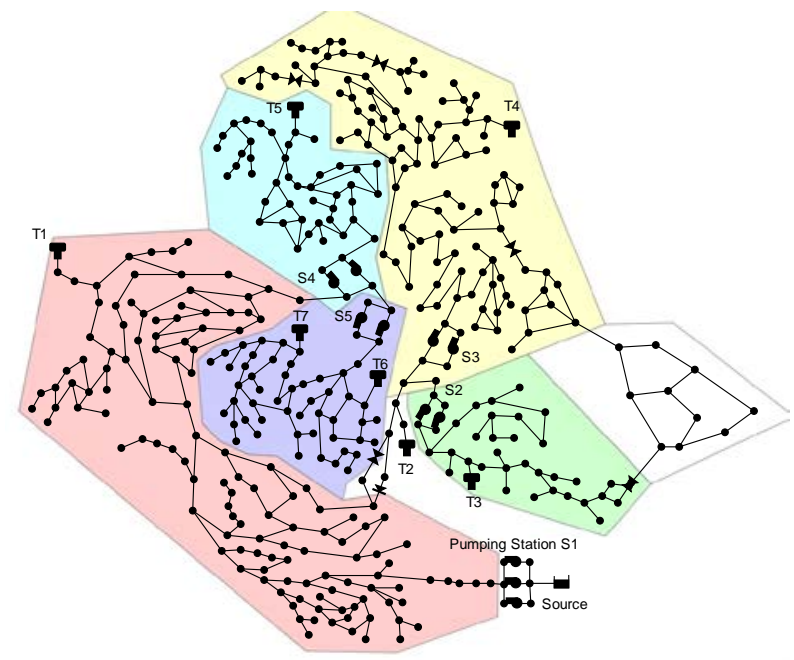

Figura 2. Modelo en EPANET de la red de distribución objeto de estudio. Proyecto año 2012.

Para ello, a lo largo de las diferentes clases se van desarrollando aspectos específicamente relacionados con la mejora de la red. A continuación se recogen estos temas y su inmediata aplicación en el proyecto:

- Análisis en régimen estático: aplicación para el estudio alternativas de funcionamiento.

- Análisis en régimen dinámico: definición del comportamiento real del sistema mediante la determinación de las consignas de operación (controles) del mismo.

- Caracterización de los consumos: representación de las pérdidas de agua en la red y estudios sobre mejora de la eficiencia hídrica del sistema. 
- Caracterización y comportamiento de depósitos: capacidad de regulación de depósitos existentes, y ampliación o redimensionado de los mismos.

- Caracterización de estaciones de bombeo: valoración energética del funcionamiento de los grupos de bombeo, selección de nuevos grupos de bombeo y mejora de la eficacia de funcionamiento de los mismos.

- Caracterización de las válvulas: ubicación de válvulas de control y regulación y determinación de sus consignas de funcionamiento.

Estos aspectos, aunque orientados por los alumnos a la resolución del proyecto planteado, constituyen el contenido fundamental de la asignatura.

De forma específica en la Tabla 1 se recoge un resumen de los elementos objeto de estudio y las diferentes opciones que sobre los mismos se han ido considerando a lo largo de los diferentes proyectos desarrollados. Como puede apreciarse cada uno de los elementos u objetos u objetos de estudio no se analizan y consideran de la misma forma en todos los proyectos. De esta forma, dependiendo del objetivo de cada año se orienta el proyecto hacia unos contenidos específicos u otros.

Tabla 1. Elementos y características definidos en cada año del proyecto.

\begin{tabular}{|c|c|c|c|c|}
\hline \multirow{2}{*}{$\begin{array}{c}\text { Elemento / Objeto } \\
\text { de estudio }\end{array}$} & \multirow{2}{*}{ Tipo de Acción } & \multicolumn{3}{|c|}{ Año del proyecto } \\
\hline & & 2012 & 2013 & 2014 \\
\hline \multirow{3}{*}{ Tuberías } & Sustituir una tubería existente & & & \\
\hline & Instalar una tubería en paralelo & & & \\
\hline & Cerrar la tubería & & & \\
\hline \multirow{4}{*}{ Bombeos } & Sustituir bomba por otra igual características & & & \\
\hline & Sustituirla bomba por otra diferente & & & \\
\hline & Rendimiento de la bomba constante & & & \\
\hline & Rendimiento de la bomba variable & & & \\
\hline \multirow{3}{*}{ Válvulas } & Cambiar la consigna la válvula & & & \\
\hline & Instalar una válvula de control & & & \\
\hline & Cerrar la válvula de control & & & \\
\hline \multirow{3}{*}{$\begin{array}{l}\text { Fugas y perdidas de } \\
\text { agua en la red }\end{array}$} & No considerar las pérdidas de agua en la red & & & \\
\hline & Pérdidas de agua constantes & & & \\
\hline & Cambiar las pérdidas al cambiar las tuberías & & & \\
\hline \multirow{2}{*}{ Calidad del agua } & Considerar la calidad del agua & & & \\
\hline & No considerar la calidad del agua & & & \\
\hline \multirow{2}{*}{$\begin{array}{l}\text { Definición de } \\
\text { sectores }\end{array}$} & No considerar sectores en la red & & & \\
\hline & Considerar los sectores definidos en la red & & & \\
\hline \multirow{3}{*}{$\begin{array}{l}\text { Controles de } \\
\text { regulación de la red }\end{array}$} & Control simples sobre elementos predefinidos & & & \\
\hline & Control simples sobre cualquier elemento & & & \\
\hline & Considerar acciones de control complejas & & & \\
\hline
\end{tabular}

\subsection{Descripción de la metodología de evaluación}

A la hora de realizar la evaluación de las diferentes soluciones se han definido una serie de criterios mediante los cuales puntuar la totalidad de los proyectos presentados. Estos criterios son:

- $\quad \mathrm{C}_{1}$ : Criterios económicos. Se valora económicamente como la suma de los costes de inversión en nuevos equipamientos (tuberías, válvulas, depósitos o bombas) y el coste explotación de la red (costes energéticos, coste del agua suministrada).

(cc) EY-NC-ND 2017, UniversitatPolitècnica de València 
- $\mathrm{C}_{2}$ : Criterios medioambientales. Se valoran mediante criterios como las tasas de emisión de $\mathrm{CO}_{2}$ o asignando un coste económico al agua consumida.

- $\quad \mathrm{C}_{3}$ : Criterios de calidad del agua. Se valora considerando el número de nudos que normalmente exceden un tiempo máximo de permanencia del agua en la red.

- $\quad \mathrm{C}_{4}$ : Criterios operativos. Son criterios como número mínimo de sectores o número máximo de arranques y paradas de un grupo de bombeo en un cierto periodo.

En primer lugar se analiza que todos los proyectos presentados cumplan las restricciones de funcionamiento impuestas (normalmente, presiones máximas y mínimas de funcionamiento, volúmenes máximos de producción, etc.). Aquellos equipos que no cumplen estas especificaciones son eliminados de la fase de concurso y su valoración deja de ser competitiva, siendo en todo caso siempre inferior a la de aquellos que cumplieron las restricciones.

Con el resto de proyectos se realiza la valoración numérica de los mismos, de forma que el proyecto i tiene en cada uno de los criterios $(\mathrm{j})$ una valoración $\mathrm{C}_{\mathrm{i}, \mathrm{j}}$. Asimismo se define para cada criterio j el valor máximo $\mathrm{C}_{\max , \mathrm{j}} \mathrm{y}$ mínimo $\mathrm{C}_{\min , \mathrm{j}}$ de entre todos los grupos que cumplen las restricciones del proyecto.

La valoración final $\mathrm{C}_{\mathrm{i}}$ de cada grupo se obtiene mediante la expresión

$$
C_{i}=\sum_{j=1}^{N_{\min }} \frac{C_{i, j}-C_{\text {min }, j}}{C_{\text {max }, j}-C_{\text {min }, j}}+\sum_{j=1}^{N_{\max }} \frac{C_{\text {max }, j}-C_{i, j}}{C_{\max , j}-C_{\text {min }, j}}
$$

donde existen dos sumandos ya que pueden existir criterios que se deseen minimizar $\left(\mathrm{N}_{\min }\right)$ y criterios que se deseen maximizar $\left(\mathrm{N}_{\max }\right)$.

De esta forma, la evaluación final de esta fase de concurso se realiza asignando la máxima calificación $\left(\mathrm{P}_{\max }=10\right.$ puntos) al equipo con menor valor del coeficiente $\mathrm{C}_{\mathrm{i}}$. Posteriormente, el profesorado realiza un análisis pormenorizado del trabajo realizado por el equipo peor clasificado (mayor valor de $\mathrm{C}_{\mathrm{i}}$ ), asignándole una calificación $\mathrm{P}_{\min }$. Finalmente la calificación $\mathrm{P}_{\mathrm{i}}$ de cada grupo se obtiene mediante la expresión

$$
\mathrm{P}_{\mathrm{i}}=\mathrm{P}_{\min }+\frac{\mathrm{C}_{\max }-\mathrm{C}_{\mathrm{i}}}{\mathrm{C}_{\max }-\mathrm{C}_{\text {min }}} \cdot\left(\mathrm{P}_{\max }-\mathrm{P}_{\min }\right)
$$

donde $\mathrm{C}_{\max }$ y $\mathrm{C}_{\min }$ son respectivamente las puntuaciones de acuerdo a la expresión (1) obtenidas por el peor y el mejor grupo.

En paralelo con la fase de concurso del trabajo el profesorado evalúa de forma conjunta tanto la memoria presentada como la presentación realizada, otorgando una calificación $\mathrm{D}_{\mathrm{i}}$. Asimismo, para garantizar el trabajo equitativo de los diferentes miembros del equipo, durante la fase de exposición el profesorado decide quién debe realizar la exposición del trabajo y quien debe contestar a cada una de las preguntas. La calificación final es una media ponderada a partes iguales entre la fase de concurso (Pi) y la calificación obtenida en la fase documental $D_{i}$. 


\section{Resultados}

La valoración de la puesta en marcha de la metodología definida en el apartado anterior se ha realizado en base a la encuesta de evaluación de la actividad del profesorado de la UPV. Dicha encuesta se imparte a los alumnos de cada asignatura al finalizar la misma y consta de una valoración global del profesorado y diversas preguntas agrupadas en cinco dominios de actuación diferentes:

- D1. Dominio de la asignatura, organización y claridad en la exposición.

- D2. Motivación del alumnado. Interacción del profesorado con el alumno.

- D3. Valoración del método de evaluación utilizado por el profesor.

- D4. Recursos utilizados.

- D5. Satisfacción general con la labor del profesor.

Tal como puede verse en la Figura 3, los resultados de las encuestas en la asignatura AMRDA desde el año 2006 hasta el año 2011 habían ido decreciendo, tanto en valor absoluto como en valores normalizados respecto de la media del MIHMA. Este hecho iba en consonancia con el aumento del número de alumnos que se inscribían en el máster. Tal como puede verse en la Figura 4, el número de alumnos inscritos en la asignatura objeto de estudio ha ido aumentando prácticamente desde la puesta en marcha de la misma hasta el año 2013 en el que se puso un límite de matrícula para la misma de 25 alumnos.
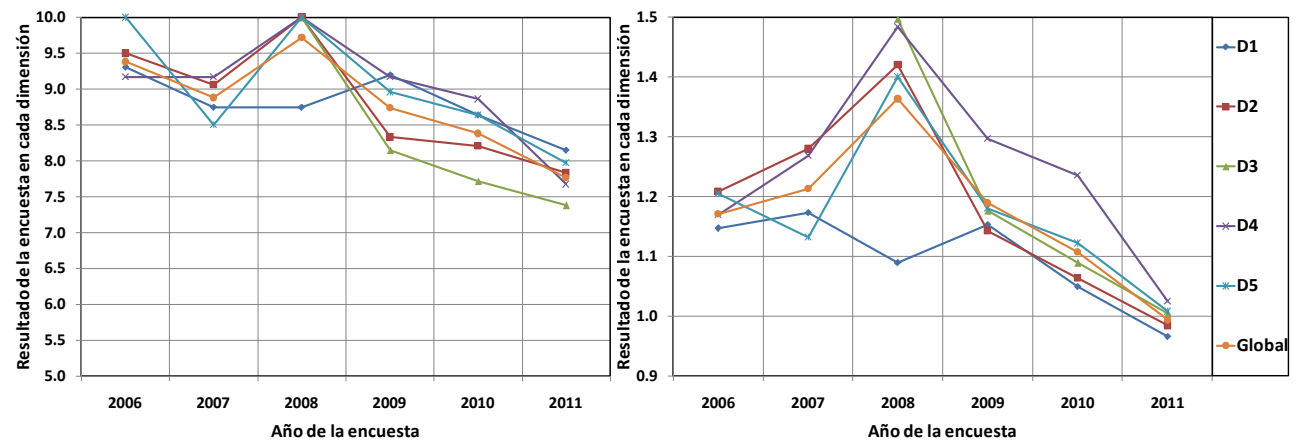

Figura 3. Resultados de las encuestas de evaluación del profesorado hasta el año 2011. En la izquierda valores absolutos. En la derecha valores normalizados con la media del MIHMA.

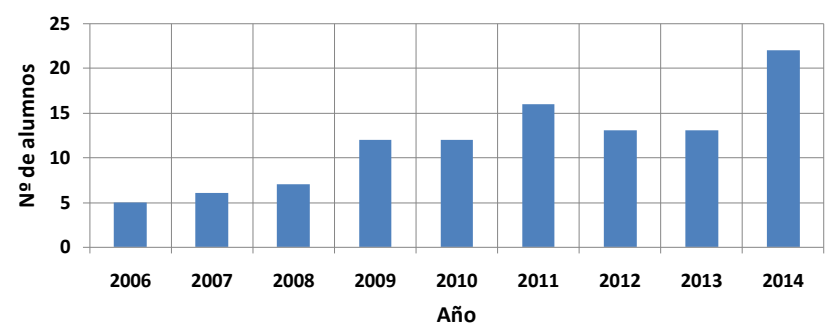

Figura 4. Evolución del número de alumnos matriculados en la asignatura AMRDA del MIHMA.

Como puede apreciarse, el análisis inicial muestra una tendencia a la disminución tanto de la motivación del alumnado (D2) como de la satisfacción global con la labor del profesor (D5). Este hecho en principio podría achacarse a que la metodología empleada hasta ese 
momento (basada en un portafolio de ejercicios) no ofrecía los resultados esperados cuando el tamaño del grupo iba aumentando.

Ante esta circunstancia se implementó para el año 2012 la metodología definida, siendo sus resultados los mostrados en la Figura 5. Como puede apreciarse, aunque el número de alumnos continuó aumentando en los años 2012-2014, todos los resultados de las encuestas mejoraron sensiblemente respecto de los valores de 2011. Más aún, manteniéndose prácticamente constante el número de alumnos en los últimos años, parece que la valoración de la motivación y valoración global del profesorado se mantengan también prácticamente constantes.
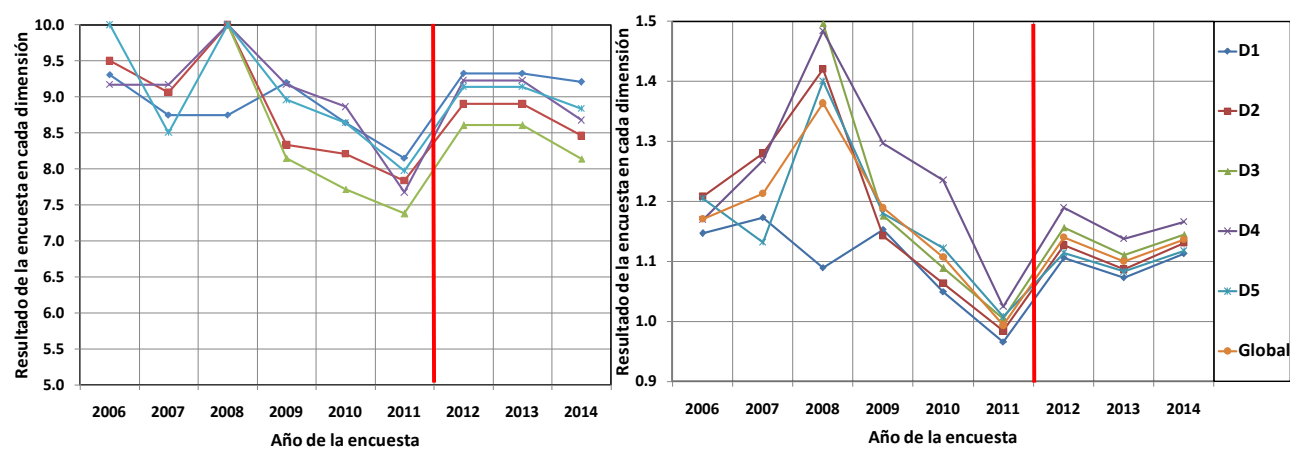

Figura 5. Resultados de las encuestas de evaluación del profesorado tras la puesta en marcha del método. En la izquierda valores absolutos. En la derecha valores normalizados con la media del MIHMA.

\section{Conclusiones}

A lo largo del presente trabajo se ha descrito una metodología de innovación educativa consistente en combinar un sistema de aprendizaje basado en proyectos con un modelo de evaluación competitivo. Tras la implantación del sistema durante tres años consecutivos, y una vez analizados los resultados, es posible extraer las siguientes conclusiones:

- Las metodologías de aprendizaje y evaluación deben estar en continua actualización y adaptarse tanto al contexto como al número de alumnos en clase. La misma metodología con el paso del tiempo suele deteriorarse, lo que requiere continuar actualizaciones.

- Algunos problemas de desafío a nivel internacional e investigador como los descritos en la Batalla de las Redes de Agua pueden ser adaptados al ámbito educativo para mejorar las dinámicas en las aulas universitarias.

- El sistema de aprendizaje basado en proyectos se ha mostrado muy flexible y capaz de adaptarse fácilmente al temario de la asignatura. Este sistema permite al alumno abordar los conocimientos desde una perspectiva práctica y muy cercana a lo que en el futuro será su realidad profesional.

- La evaluación competitiva ha supuesto sin duda una enorme mejora tanto en los resultados de la valoración del profesorado como en la motivación por parte de los alumnos. Aunque los datos empíricos de las encuestas no lo recojan, se ha detectado un notable interés de los alumnos por la asignatura. De hecho, su 
intensidad en la dedicación al trabajo sobre la misma ha aumentado fomentando en muchas ocasiones la curiosidad por explorar ámbitos de conocimiento más allá de los contenidos de la materia objeto de estudio.

El sistema definido se ha mostrado exitoso tal como ponen de manifiesto los resultados de las encuestas realizadas. No obstante, dicho método puede irse adaptando más poco a poco para aumentar dinámicas de grupo como el liderazgo o la formación colaborativa entre grupos.

\section{Referencias}

ABT, C. (1970). Serious games. New York: Viking Press.

ADIN MARCOS, I., BERENGUER PÉREZ, R., BISTUÉ GARCÍA, G., GUTIÉRREZ GARCÍA, I., \& MENDIZABAL SAMPER, J. (2010). Introducing CAD Tools for Electronic Design Using a Competition Based Evaluation Method. IX Congreso del Tecnologías Aplicadas a la Enseñanza de la Electrónica - TAEE, pp. 641-697. Madrid, noviembre 2010.

ÁLVAREZ, B. Á., MIERES, C. G., y RODRÍGUEZ, N. G. (2008). La motivación y los métodos de evaluación como variables fundamentales para estimular el aprendizaje autónomo. Revista de Docencia Universitaria, 1(2).

BABA, A.F., CIN, F.M., \& BAKANAY, D. (2012). A fuzzy system for evaluating students' project in engineering education. Computer Applications in Engineering Education, 20(2), 287-294.

BERIAin, A., DEL RIO, D., SOlAR, H., AlVARADO, U., \& DEL PORTILlO, J. (2014). Challenge oriented methodology for analog integrated circuit layout design training. In Tecnologias Aplicadas a la Ensenanza de la Electronica (Technologies Applied to Electronics Teaching)(TAEE), 2014 XI (pp. 1-5). IEEE.

BOURGAULT, M., \& LAGACE, D. (2002). A seminar for real-time interactive simulation of engineering projects: An innovative use of video-conferencing and IT-based educational tools. Journal of Engineering Education, 91(2), 177.

CARO, M. I., GONZÁLEZ, S. Á., y RUBIO, R. Á. (2008). Métodos de evaluación en la enseñanza superior. Revista de Investigación educativa, 26(2), 539-552.

CARPENTER, S.L., DELUGACH, H.S., ETZKORN, L.H., FARRINGTON, P.A., FORTUNE, J.L., UTLEY, D.R., \& VIRANI, S.S. (2007). A knowledge modeling approach to evaluating student essays in engineering courses. Journal of Engineering Education, 96(3), 227-239.

CHUMBLEY, L. S., HARGRAVE, C. P., CONSTANT, K., \& HAND, B. (2002). Project ExCEL: Web-based scanning electron microscopy for K-12 education. Journal of Engineering Education, 91(2), 203. 
CERATO, A., ELTON, D., \& SHANNON, D. (2011). Building student teamwork with the student geo-challenge. Journal of Professional Issues in Engineering Education \& Practice, 138(1), 14-20.

CLARKE, B. G. (2007). Introducing students to professional practice in civil engineering. Journal of Professional Issues in Engineering Education and Practice, 133(2), 107-115.

ÇULHA, D. (2016). Applying competition-based learning to agile software engineering. Computer Applications in Engineering Education, 24(3), 382-387.

DAVIDOVITCH, L., PARUSH, A., \& SHTUB, A. (2006). Simulation-based Learning in Engineering Education: Performance and Transfer in Learning Project Management. Journal of Engineering Education, 95(4), 289-299.

DE LOS RIOS, I., CAZORLA, A., DÍAZ-PUENTE, J. M., \& YAGÜE, J. L. (2010). Project-based learning in engineering higher education: two decades of teaching competences in real environments. Procedia-Social and Behavioral Sciences, 2(2), 1368-1378.

DUKE, R. D. (1974). Gaming: the future's language (1st ed., p. 223). New York: Sage Publications.

GADHAMSHETTY, V., SHRESTHA, N., \& KILDUFF, J. E. (2016). Project-Based Introduction to an Engineering Design Course Incorporating Microbial Fuel Cells as a Renewable Energy Technology. Journal of Professional Issues in Engineering Education and Practice, 142(3), 05016001.

GIUSTOLISI, O., BERARDI, L., LAUCELLI, D., SAVIC, D., \& KAPELAN, Z. (2015). Operational and tactical management of water and energy resources in pressurized systems: Competition at WDSA 2014. Journal of Water Resources Planning and Management, 142(5), C4015002.

GONZÁLEZ-MARCOS, A., ALBA-ELÍAS, F., ORDIERES-MERÉ, J., \& NAVARIDASNALDA, F. (2014). Cross-analysis of Transversal Competences in Project Management. In CSEDU (2) (pp. 34-41).

GUZMÁN-RAMÍREZ, E., \& GARCIA, I. A. (2013). Using the project-based learning approach for incorporating an FPGA-based integrated hardware/software tool for implementing and evaluating image processing algorithms into graduate level courses. Computer Applications in Engineering Education, 21(S1), E73-E88.

HENRY, W. P. (2002). Professional issues in civil engineering in the 21st century. Journal of Professional Issues in Engineering Education and Practice, 128(4), 160-161.

HERNANDEZ-LINARES, R., AGUDO, J. E., RICO, M., \& SÁNCHEZ, H. (2015). Transversal competences of university students of engineering. Croatian Journal of Education17(2), 383-409.

IGLESIAS-REY, P.L., MARTÍNEZ-SOLANO, F.J., MORA MELIÁ, D., \& MARTÍNEZSOLANO, P.D. (2015). Combining Engineering Judgment and an Optimization Model 
to Increase Hydraulic and Energy Efficiency in Water Distribution Networks. Journal of Water Resources Planning and Management, 142(5), C4015012.

ILIE-ZUDOR, E., KEMÉNY, Z., SZATHMÁRI, M., \& MONOSTORI, L. (2013). The role of OSC-based projects in meeting new challenges of education-concept and exemplification. Computer Applications in Engineering Education, 21(S1), E141-E151.

LABOSSIÈRE, P., \& BISBY, L. A. (2009). Lessons learned from a design competition for structural engineering students: The case of a pedestrian walkway at the Universite de Sherbrooke. Journal of Professional Issues in Engineering Education and Practice, 136(1), 48-56.

LUCE, L., \& HENRY, W. P. (2002). Public awareness of civil engineering and the infrastructure. Journal of Professional Issues in Engineering Education and Practice, 128(4), 165-166.

MAHENDRAN, M. (1995). Project-based civil engineering courses. Journal of Engineering Education, 84(1), 75-79.

MARCHI, A., SALOMONS, E., OSTFELD, A., KAPELAN, Z., SIMPSON, A. R., ZECCHIN, A. C., ... \& WALSKI, T. (2013). Battle of the water networks II. Journal of water resources planning and management, 140(7), 04014009.

MAYER, I., BEKEBREDE, G., HARTEVELD, C., WARMELINK, H., ZHOU, Q., RUIJVEN, T., ... \& WENZLER, I. (2014). The research and evaluation of serious games: Toward a comprehensive methodology. British Journal of Educational Technology, 45(3), 502-527.

MERINO, D. N., \& ABEL, K. D. (2003). Evaluating the Effectiveness of Computer Tutorials Versus Traditional Lecturing in Accounting Topics. Journal of Engineering Education, 92(2).

MORLEY, M. S., KHOURY, M., \& SAVIC, D. (2016). Serious Game Approach to Water Distribution System Design and Rehabilitation Problems. 14th International Conference on Computing and Control in the Water Industry - CCWI 2016, At Amsterdam, Netherlands.

OSTFELD, A., UBER, J. G., SALOMONS, E., BERRY, J. W., HART, W. E., PHILLIPS, C. A., ... \& DI PIERRO, F. (2008). The battle of the water sensor networks (BWSN): A design challenge for engineers and algorithms. Journal of Water Resources Planning and Management, 134(6), 556-568.

OSTFELD, A., SALOMONS, E., ORMSBEE, L., UBER, J. G., BROS, C. M., KALUNGI, P., ... \& LANSEY, K. (2011). Battle of the water calibration networks. Journal of Water Resources Planning and Management, 138(5), 523-532.RESSLER, S. J., \& RESSLER, E. K. (2004). Using a nationwide Internet-based bridge design contest as a vehicle for engineering outreach. Journal of Engineering Education, 93(2), 117. 
ROMERO, C., VENTURA, S., \& DE BRA, P. (2009). Using mobile and web-based computerized tests to evaluate university students. Computer Applications in Engineering Education, 17(4), 435-447.

ROSSMAN, L. A. (2000). EPANET 2: user's manual. EPA/600/R- 00/057, Water Supply and Water Resources Division, National Risk Management Research Laboratory, Office of Research and Development, U.S. EPA, Cincinnati.

SANCHEZ, A., CANNON-BOWERS, J. \& BOWERS, C. (2010). Establishing a science of game based learning. In J. A. Cannon-Bowers \& C. Bowers (Eds.), Serious game design and development: technologies for training and learning (pp. 290-304). Hershey, PA: IGI Global. doi:10.4018/978-1-61520-739-8.ch016.

SAVIC, D. A., MORLEY, M. S., \& KHOURY, M. (2016). Serious Gaming for Water Systems Planning and Management. Water, 8(10), 456.

SCHAFFER, S. P., CHEN, X., ZHU, X., \& OAKES, W. C. (2012). Self-Efficacy for Cross-Disciplinary Learning in Project-Based Teams. Journal of Engineering Education, 101(1), 82-94.

SEE, A. (2006). Challenging computer-based projects for a Mechatronics course: Teaching and learning through projects employing virtual instrumentation. Computer Applications in Engineering Education, 14(3), 222-242.

SHYR, W. J. (2012). Teaching mechatronics: An innovative group project-based approach. Computer Applications in Engineering Education, 20(1), 93-102.

SLAVIN, R. \& \& JOHNSON, R. T. (1999). Aprendizaje cooperativo: teoría, investigación y práctica. Buenos Aires: Aique.

TRAVIS, J., \& KRING, J. (2006). LabVIEW for everyone: Graphical programming made easy and fun (National instruments virtual instrumentation series). Prentice Hall PTR.

URAL, A. (2013). A hands-on finite element modeling experience in a multidisciplinary project-based freshman course. Computer Applications in Engineering Education, 21(2), 294-299.

VALDIVIA, I.Á. (2008). Evaluación del aprendizaje en la universidad: una mirada retrospectiva y prospectiva desde la divulgación científica. Electronic Journal of Research in Educational Psychology, 6(14), 235-271.

WALSKI, T. M., BRILL JR, E. D., GESSLER, J., GOULTER, I. C., JEPPSON, R. M., LANSEY, K., ... \& ORMSBEE, L. (1987). Battle of the network models: Epilogue. Journal of Water Resources Planning and Management, 113(2), 191-203.

WANKAT, P. C. (2005). Undergraduate student competitions. Journal of Engineering Education, 94(3), 343.

WU, Y. (2012). Courseware design of project research based on interactive game design. Computer Applications in Engineering Education, 20(3), 510-517. 
Combinación de una metodología basada en proyectos y de una evaluación competitiva en la asignatura Análisis y modelación de redes de distribución de agua

ZAVALANI, O. (2015). Computer-based simulation development of a design course project in electrical engineering. Computer Applications in Engineering Education, 23(4), 587-595.

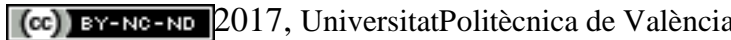

